\title{
ALTERNATING FORMS AND ONE-RELATOR GROUPS
}

\author{
JACK SONN
}

ABSTRACT. This paper considers epimorphisms of finitely generated one-relator groups $G=F / R, F$ free, $R \subseteq F p[F, F]$ for some rational prime $p$. The main result is a lower bound for the difference rank $G$ rank $G^{\prime}$ when $G^{\prime}$ is a one-relator group homomorphic to $G$. This generalizes a known result for the case $G^{\prime}$ a free group.

Let $V$ be a finite-dimensional vector space over a field $F$ equipped with an alternate bilinear form $(X, Y) \mapsto X Y \in F ; X, Y \in V(X X=0$ for every $X \in V)$. Given a subset $S$ of $V$ let $S^{*}$ denote the subspace $\{X \in V \mid X Y=0$ for all $Y \in S\}$. A subspace $W$ of $V$ is called isotropic if $X Y=0$ for all $X, Y \in W$. The radical $V^{*}$ of $V$ is denoted by $\operatorname{rad} V$ and is clearly isotropic. If $W$ is a subspace of $V$ then $\operatorname{rad} W=W \cap W^{*} . W$ is nonsingular if $\operatorname{rad} W=0$.

Lemma 1. Let $V$ be a finite-dimensional space over a field $F$ equipped with an alternate bilinear form $(X, Y) \rightarrow X Y \in F$, and let $U$ be a subspace of $V$. Then $U$ has a direct sum decomposition

$$
U=U_{1} \oplus U_{0} \oplus(\operatorname{rad} U \cap \operatorname{rad} V),
$$

where $U_{0} \oplus(\operatorname{rad} U \cap \operatorname{rad} V)=\operatorname{rad} U$, and $U_{1}$ is nonsingular. Furthermore, this decomposition can be extended to a decomposition

$$
V=V_{1} \oplus U_{1} \oplus U_{0} \oplus U_{0}^{\prime} \oplus(\operatorname{rad} U \cap \operatorname{rad} V) \oplus V_{0},
$$

where $U_{0}^{\prime}$ is an isotropic subspace of $V$ of the same dimension as $U_{0}$ and $U_{0} \oplus U_{0}^{\prime}$ is nonsingular, $V_{1}$ is nonsingular, and $\operatorname{rad} V=(\operatorname{rad} U \cap \operatorname{rad} V)$ $\oplus V_{0}$.

Proof. By [1, Theorem 3.3, p. 116], $U=U_{1} \oplus \operatorname{rad} U, U_{1}$ nonsingular. Write $\operatorname{rad} U=(\operatorname{rad} U \cap \operatorname{rad} V) \oplus U_{0}, \operatorname{rad} V=(\operatorname{rad} U \cap \operatorname{rad} V) \oplus V_{0} ; U_{0}, V_{0}$ subspaces of $V$. Since $U_{0} \cap \operatorname{rad} V=0, U+\operatorname{rad} V$ is the direct sum

Received by the editors August 6, 1973.

AMS (MOS) subject classifications (1970). Primary 20F05, 20 J05, 20E 15;

Secondary $20 \mathrm{E} 40,20 \mathrm{~F} 20$.

Key words and phrases. Alternating forms, one-relator groups, cohomology, factor groups, nilpotent groups, rank. 
$U_{1} \oplus U_{0} \oplus \operatorname{rad} V$. Write $V=V_{1}^{\prime} \oplus U_{1} \oplus U_{0} \oplus \operatorname{rad} V$. Then $V_{2}=V_{1}^{\prime} \oplus U_{1} \oplus U_{0}$ is non singular. By [1, Theorem 3.5, p. 117], $V_{2}=U_{1} \oplus\left(U_{1}^{*} \cap V_{2}\right)$, and $U_{1}^{*} \cap V_{2}$ is nonsingular. Also $U_{1}^{*} \cap V_{2} \supseteq U_{0}$. By [1, Theorem 3.8, p. 120] there exists an isotropic subspace $U_{0}^{\prime}$ of (the nonsingular space) $U_{1}^{*} \cap V_{2}$ such that $U_{0} \oplus U_{0}^{\prime}$ is nonsingular. Set $V_{1}=\left(U_{0} \oplus U_{0}^{\prime}\right)^{*} \cap\left(U_{1}^{*} \cap V_{2}\right)$. We may now write $U_{1}^{*} \cap V_{2}=V_{1} \oplus U_{0} \oplus U_{0}^{\prime}$, and finally, $V=V_{2} \oplus \operatorname{rad} V=V_{1}$ $\oplus U_{0} \oplus U_{0}^{\prime} \oplus U_{1} \oplus(\operatorname{rad} U \cap \operatorname{rad} V) \oplus V_{0}$. Q.E.D.

Let $F$ be the free group on $n$ generators. Let $p$ be an odd rational prime and define the $p$-central series recursively: $F_{0}=F, F_{m}=$ $F_{m-1}^{p}\left[F, F_{m-1}\right]$, where $F_{m-1}^{p},\left[F, F_{m-1}\right]$ denote the subgroups generated by $p$ th powers of elements of $F_{m-1}$, and the commutators of elements of $F_{m-1}$ with elements of $F$, respectively. Let $G=F / R$, where $R$ is the normal subgroup of $F$ generated by an element $r \in F_{1}$. Let $F^{\prime}$ be the free group on $m \leq n$ generators, $G^{\prime}=F^{\prime} / R^{\prime}, R^{\prime}$ an arbitrary normal subgroup of $F^{\prime}$ contained in $F_{1}^{\prime}$ (where $\left\{F_{i}^{\prime}\right\}$ is defined analogous to $\left\{F_{i}\right\}$ ).

Suppose there is an epimorphism $f: G \rightarrow G^{\prime} . f$ induces a monomorphism $f^{*}: H^{1}\left(G^{\prime}\right) \rightarrow H^{1}(G)$ where $H^{i}(G), H^{i}\left(G^{\prime}\right)$ denote the $i$ th cohomology groups $H^{i}(G, \mathbf{Z} / p \mathbf{Z}), H^{i}\left(G^{\prime}, \mathbf{Z} / p \mathbf{Z}\right)$, respectively, over $\mathbf{Z} / p \mathbf{Z}$, with trivial action on $\mathbf{Z} / p \mathbf{Z}$. We have a commutative diagram

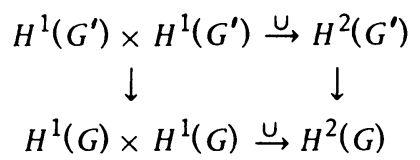

where $U$ denotes the cup product, and the vertical arrows are induced by $f$. One can show that $H^{2}(G) \simeq \mathrm{Z} / p \mathbf{Z}$ so that the cup product makes $V=H^{1}(G)$ an $n$-dimensional $\mathbf{Z} / p \mathbf{Z}$-space equipped with an altemate bilinear form. (For details, see [2].) We set $U=f^{*} H^{1}\left(G^{\prime}\right)$, which is a subspace of $V$.

It is shown in [2] that the mapping $\bar{r}: H^{2}(G) \rightarrow \mathrm{Z} / p \mathbf{Z}$ defined by $\bar{r}(a)=\operatorname{tg}^{-1}(a)\left(r^{-1}\right) \in \mathbf{Z} / p \mathbf{Z}, a \in H^{2}(G)$, where $\operatorname{tg}: H^{1}(R)^{F} \rightarrow H^{2}(G)$ is the transgression map, is an isomorphism. The bilinear form induced on $V$ by the cup product will be defined to be $\bar{r}\left(\chi \cup \chi^{\prime}\right), \chi, \chi^{\prime} \in V$. We will henceforth write $\chi \chi^{\prime}=\bar{r}\left(\chi \cup \chi^{\prime}\right)$.

We now apply Lemma 1 and obtain a direct sum decomposition

$$
V=V_{1} \oplus U_{1} \oplus U_{0} \oplus U_{0}^{\prime} \oplus(\operatorname{rad} U \cap \operatorname{rad} V) \oplus V_{0} \text {, }
$$

where $(\operatorname{rad} U \cap \operatorname{rad} V) \oplus V_{0}=\operatorname{rad} V$, $V_{1}, U_{1}, U_{0} \oplus U_{0}^{\prime}$ nonsingular, $U=U_{1} \oplus \operatorname{rad} U, \operatorname{rad} U=U_{0} \oplus(\operatorname{rad} U \cap \operatorname{rad} V)$, 
$U_{0}, U_{0}^{\prime}$ isotropic of the same rank. By [1, Theorem 3.7, p. 119] there is a basis $\alpha_{1}, \cdots, a_{2 l}$ of $V_{1}$ such that $a_{2 i-1} \alpha_{2 i}=1$ for $1 \leq i \leq l, a_{i} a_{j}=$ 0 for $i<j$ otherwise, $1 \leq i, j \leq 2 l$.

Similarly, there is a basis $\beta_{1}, \cdots, \beta_{2 s}$ of $U_{1}$ such that $\beta_{2 i-1} \beta_{2 i}=$ 1 , etc. By [1, Theorem 3.8] there are bases $\gamma_{1}, \gamma_{3}, \cdots, \gamma_{2 t-1}$ of $U_{0}$, and $\gamma_{2}, \gamma_{4}, \cdots, \gamma_{2 t}$ of $U_{0}^{\prime}$ such that $\gamma_{2 i-1} \gamma_{2 i}=1,1 \leq i \leq t, \gamma_{i} \gamma_{j}=0$ otherwise, $1 \leq i<j \leq 2 t$. Finally we take arbitrary bases $\delta_{1}, \cdots, \delta_{k}$ of $\operatorname{rad} U$ $\cap \operatorname{rad} V$ and $\rho_{1}, \cdots, \rho_{q}$ of $V_{0}$.

Let $\left\{\chi_{1}, \cdots, \chi_{n}\right\}$ be the union of all the bases defined below. It is clearly a basis of $V$. Similarly, let $\eta_{1}, \cdots, \eta_{m}$ be the basis of $H^{1}\left(G^{\prime}\right)$ such that $\left\{f^{*}\left(\eta_{1}\right), \cdots, f^{*}\left(\eta_{m}\right)\right\}$ is the union of the bases of $U_{0}, U_{1}$, and $\operatorname{rad} U \cap \operatorname{rad} V$ given below.

We may lift $f$ to a homomorphism $f^{\prime}: F \rightarrow F^{\prime}$ such that the diagram

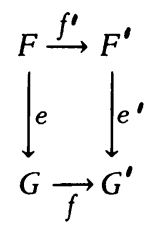

commutes, where $e, e^{\prime}$ are the canonical mappings. For let $z_{1}, \cdots, z_{n}$ be a basis of $F$. We may choose $u_{1}, \cdots, u_{n} \in F^{\prime}$ such that $f e\left(z_{i}\right)=e^{\prime}\left(u_{i}\right)$, $1 \leq i \leq n$, and there is a unique homomorphism $f^{\prime}: F \rightarrow F^{\prime}$ such that $f^{\prime}\left(z_{i}\right)=u_{i}, 1 \leq i \leq n$ (a basic property of free groups). There is induced a commutative diagram

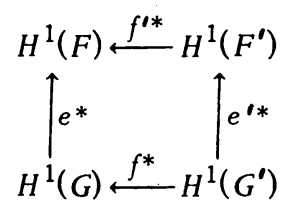

by means of which we identify $H^{1}(F)$ with $H^{1}(G), H^{1}\left(F^{\prime}\right)$ with $H^{1}\left(G^{\prime}\right)$, and hence $f^{*}$ with $f^{\prime *}$. (Note that $f^{\prime}(F) F_{1}^{\prime}=F^{\prime}$, hence, $f^{\prime *}$ is monomorphic.) We utilize these identifications in the following discussion.

By duality there exist $x_{1}, \cdots, x_{n} \in F$ such that $\chi_{i}\left(x_{j}\right)=\delta_{i j}, 1 \leq i, j$ $\leq n$, and $y_{1}, \cdots, y_{m} \in F^{\prime}$ such that $\eta_{i}\left(y_{j}\right)=\delta_{i j}, 1 \leq i, j \leq m$. These are not necessarily bases of $F$ and $F^{\prime}$; however, by Burnside's basis theorem, they do map onto bases of $F / F_{2}$ and $F^{\prime} / F_{2}^{\prime}$ respectively.

Now for $1 \leq i \leq m, 1 \leq j \leq n, \eta_{i} f^{\prime}\left(x_{j}\right)=f^{*}\left(\eta_{i}\right)\left(x_{j}\right)=\chi_{k(i)}\left(x_{j}\right)=\delta_{k(i), j}$ for some $k(i), 1 \leq k(i) \leq n$, where $\chi_{k(i)}=f^{*}\left(\eta_{i}\right) \in U$. If $j \notin\{k(i): 1 \leq i \leq m\}$, 
then $\eta_{i} f^{\prime}\left(x_{j}\right)=0$ for $1 \leq i \leq m$, hence $f^{\prime}\left(x_{j}\right) \equiv 1\left(\bmod F_{1}^{\prime}\right)$. On the other hand, if $j=k(t)$ for some $t, 1 \leq t \leq m$, then $\eta_{i} f^{\prime}\left(x_{j}\right)=\delta_{k(i), k(t)}=\delta_{i t}=$ $\eta_{i}\left(y_{t}\right)$ for $1 \leq i \leq m$, hence $f^{\prime}\left(x_{j}\right) \equiv y_{t}\left(\bmod F_{1}^{\prime}\right)$.

By [2, Proposition 1], the relator $r$ satisfies the congruence

$$
r \equiv \prod_{i=1}^{n} x_{i}^{p a_{i}} \prod_{i=1}^{l+s+t}\left[x_{2 i-1}, x_{2 i}\right] \quad\left(\bmod F_{2}\right)
$$

where $a_{i} \in \mathbf{Z} / p \mathbf{Z}$, and $\left\{\chi_{1}, \cdots, \chi_{2 l+2 s+2 t}\right\}=\left\{\alpha_{1}, \cdots, \alpha_{2 l}, \beta_{1}, \cdots, \beta_{2 s}\right.$, $\left.\gamma_{1}, \cdots, \gamma_{2 t}\right\}$ in that order. It follows that

$$
f^{\prime}(r) \equiv \prod_{i=1}^{m} y_{i}^{p b} \cdot \prod_{i=1}^{s}\left[y_{2 i-1}, y_{2 i}\right] \quad\left(\bmod F_{2}^{\prime}\right)
$$

where $b_{i}=a_{k(i)}, \chi_{k(i)}=f^{*}\left(\eta_{i}\right)$, and $\left\{\beta_{1}, \cdots, \beta_{2 s}\right\}=\left\{f^{*}\left(\eta_{1}\right), \cdots, f^{*}\left(\eta_{2 s}\right)\right\}$ in that order. We have therefore proved the following theorem.

Theorem 1. Let $G$ be a finitely generated one-relator group $F / R, F$ free of rank $n, R$ the normal subgroup of $F$ generated by an element $r \in F_{1}=F^{p}[F, F], p$ an odd rational prime. Suppose $f$ is an epimorphism of $G$ onto a group $G^{\prime}=F^{\prime} / R^{\prime}$, where $F^{\prime}$ is free on $m \leq n$ generators and $R^{\prime} \leq F_{1}^{\prime}$. Then there exist $x_{1}, \cdots, x_{n} \in F, y_{1}, \cdots, y_{m} \in F^{\prime}$, which map onto bases of $F / F_{2}, F^{\prime} / F_{2}^{\prime}$ respectively $\left(F_{2}=F_{1}^{p}\left[F_{1}, F\right]\right)$, and nonnegative integers $l, s, t, k, q$, such that $2 l+2 s+2 t+k+q=n, 2 s+t+k=m$, such that $r$ takes the form (2) above $\left(\bmod F_{2}\right)$, and $f$ lifts to a homomorphism $f^{\prime}: F \rightarrow F^{\prime}$ such that all into $F_{1}^{\prime}$;

(i) $f^{\prime}$ maps $x_{1}, \cdots, x_{2 l} ; x_{2(l+s+1)}, \cdots, x_{2(l+s+t)} ; x_{n-q+1}, \cdots, x_{n}$

(ii) $f^{\prime}$ maps $x_{2 l+1}, \cdots, x_{2 l+2 s} ; x_{2(l+s)+1}, x_{2(l+s)+3}, \cdots, x_{2(l+s+t)-1}$; $x_{2(l+s+t)+1}, \cdots, x_{2(l+s+t)+k}$, onto $y_{1}, \cdots, y_{m}\left(\bmod F_{2}^{\prime}\right)$, in the given order.

There are two special cases of particular interest. The first is when $G^{\prime}$ is free or even when $R^{\prime} \leq F_{2}^{\prime}$. In this instance $s=0$ since $f^{\prime}(r) \in R^{\prime}$, and for each pair $\left\{x_{2 i-1}, x_{2 i}\right\}, 1 \leq i \leq l+t, f^{\prime}$ either maps both into $F_{1}^{\prime}$ or $x_{2 i-1}$ onto a $y_{j}$ and $x_{2 i}$ into $F_{1}^{\prime}$. The remaining $x_{i}$ map either onto a $y_{j}$ or into $F_{1}^{\prime}$. Furthermore, $m=t+k$ and $n=2 l+2 t+k+q$, hence $n-m$ $=2 l+t+q \geq l+t$. Hence in general if $G^{\prime}$ is free, then

$$
\text { rank } G^{\prime} \leq \operatorname{rank} G-\rho / 2 \text {, }
$$

where $\rho=2(l+t)$ is the dimension of $V / \mathrm{rad} V$. This inequality is obtained in [2]. 
The second case of interest is when $G^{\prime}$ is a one-relator group, $R^{\prime}$ being the normal subgroup of $F^{\prime}$ generated by an el ement $r^{\prime} \in F_{1}^{\prime}$. Since $f^{\prime}(r) \in R^{\prime}, f^{\prime}(r) \equiv r^{b}\left(\bmod F_{2}^{\prime}\right)$ for some $b \in \mathbf{Z}, 0 \leq b \leq p-1$. Furthermore,

$$
\begin{aligned}
n-m & =(2 l+2 s+2 t+k+q)-(2 s+t+k)=2 l+t+q \\
& \geq 2 l+t \geq l+t=1 / 2\left(\rho(G)-\rho\left(G^{\prime}\right)\right),
\end{aligned}
$$

where $\rho(G)=\operatorname{dim}(V / \operatorname{rad} V)$ and $\rho\left(G^{\prime}\right)=\operatorname{dim}(U / \operatorname{rad} U)$. We thus have the following theorem.

Theorem 2. Let $G=F / R, G^{\prime}=F^{\prime} / R^{\prime}$ be (one-relator) presentations of one-relator groups $G, G^{\prime}$ such that $R \leq F_{1}, R^{\prime} \leq F_{1}^{\prime}$. If there is an epimorphism of $G$ onto $G^{\prime}$, then

$$
\text { rank } G-\operatorname{rank} G^{\prime} \geq 1 / 2\left(\rho(G)-\rho\left(G^{\prime}\right)\right),
$$

where $\rho(G)=\operatorname{dim}\left(H^{1}(G, \mathbf{Z} / p \mathbf{Z}) / \operatorname{rad} H^{1}(G, \mathbf{Z} / p \mathbf{Z})\right), \rho\left(G^{\prime}\right)$ defined similarly, where $H^{1}(G, \mathbf{Z} / p \mathbf{Z}), H^{1}\left(G^{\prime}, \mathbf{Z} / p \mathbf{Z}\right)$ are considered as $\mathbf{Z} / p \mathbf{Z}$-spaces with an alternate bilinear form given by the cup product. (Note. We have utilized the commutativity of diagram (1) to obtain $\left.\rho\left(G^{\prime}\right)=\operatorname{dim}(U / \operatorname{rad} U).\right)$

Remarks. 1. By the proof of Theorem $1, \rho(G)$ can al so be defined as the number of $x_{i}$ occuring in the "canonical" form $\left(\bmod F_{2}\right)$ $\Pi_{i=1}^{\alpha(G) / 2}\left[x_{2 i-1}, x_{2 i}\right]$ of a relator $r$ defining $G$.

2. In equality (4) can be looked upon as a generalization of (3) since if $G^{\prime}$ is free, $\rho\left(G^{\prime}\right)=0$.

3. Suppose $r \in[F, F]$, the commutator subgroup of $F$. Then $r \in F_{1}=$ $F^{p}[F, F]$ for every $p$. It is shown in [2] that there is a basis $x_{1}, \cdots, x_{n}$ of $F$ such that

$$
r \equiv \prod_{i=1}^{n^{\prime}}\left[x_{2 i-1}, x_{2 i}\right]^{a} \quad\left(\bmod F_{2}^{(0)}\right),
$$

where $F_{2}^{(0)}=[F,[F, F]]$ and $0<a_{i} \in \mathbf{Z}, 2 n^{\prime} \leq n$, and that $\rho_{0}=\rho_{0}(G)=2 n^{\prime}$ is the rank of the cup product $H^{1}(G, \mathbf{Z}) \times H^{1}(G, \mathbf{Z}) \rightarrow H^{2}(G, \mathbf{Z}) \simeq \mathbf{Z}$ as a $\mathbf{Z}$ bilinear form. If we choose $p$ so that $p$ does not divide any of the $a_{i}$, then reducing $r \bmod F_{2}\left(\supset F_{2}^{(0)}\right)$ yields $r \equiv \Pi_{i=1}^{n^{\prime}}\left[x_{2 i-1}, x_{2 i}^{\left.a_{i}\right]}\left(\bmod F_{2}\right)\right.$, a "canonical" form for $r \bmod F_{2}$, hence by Remark $1, \rho(G)=\rho_{0}(G)$, where $\rho(G)$, as defined above, is the rank of the cup product on $H^{1}(G, \mathbf{Z} / p \mathbf{Z})$. Thus (3) yields an in equality due to A. Steinberg [2], and (4) the corresponding generalization of it.

4. Let $f_{1}$ be an epimorphism from $F / R F_{2}$ onto $F^{\prime} / R^{\prime} F_{2}^{\prime}$, where $F, R$, $F^{\prime}, R^{\prime}$ are as in Theorem 1. $f_{1}$ lifts to an $f^{\prime}: F \rightarrow F^{\prime}$ by the same argu- 
ment used in lifting $f$ to $f^{\prime}$. By utilizing the mapping $f: F / R \rightarrow F^{\prime} / R^{\prime} F_{2}^{\prime}$ induced by $f^{\prime}$, and the proof of Theorem 1 , we obtain a version of Theorem 1 with $f$ replaced by $f_{1}$. On the other hand, every $f$ induces an $f_{1}$, so the new version yields the original as well. Similarly, in Theorem 2, if we observe that the only assertions about $f^{\prime}$ that are made are about the composite of $f^{\prime}$ with the canonical mapping $F^{\prime} \rightarrow F^{\prime} / F_{2}^{\prime}$, we obtain an equivalent version of Theorem 2 with $f$ replaced by $f_{1}: F / R F_{2} \rightarrow F^{\prime} / R^{\prime} F_{2}^{\prime}$.

This last remark, together with Remark 3, yields (the apparently stronger version of) the in equality rank $G^{\prime} \leq \operatorname{rank} G-\rho_{0}(G) / 2$ when $G, G^{\prime}$ are nilpotent of class 2 (or $c>2$ ) and $r \in[F, F]$, which Steinberg [3] proves using other methods.

5. Theorems 1 and 2 and their corollaries can be proved in the category of pro-p-groups. (Here one allows only continuous homomorphisms and closed subgroups.) The arguments are completely analogous and even simpler and more elegant, by virtue of the Burnside basis theorem which holds for pro-p-groups [4, Chapter I]. The necessary cohomology is developed in [5]. In particular, the sets $\left\{x_{1}, \cdots, x_{n}\right\}$ and $\left\{y_{1}, \cdots, y_{m}\right\}$ which appear in Theorem 1 are actual bases of $F$ and $F^{\prime}$. If $F(p)$ is the free pro-p-group on $n$ generators, then the (finite) factor groups $F / F_{2}$ (in fact $F / F_{c}$ ) and $F(p) / F(p)_{2}$ (respectively $\left.F(p) / F(p)_{c}\right)$ are isomorphic, as well as $F / R F_{2}$ and $F(p) / \bar{R} F(p)_{2}$, where $\bar{R}$ is the closed normal subgroup of $F(p)$ corresponding to $R$. (One can assume, if one wishes, that $F$ is embedded in $F(p)$; then $\bar{R}$ is the (topological) closure of $R$ in $F(p)$.) Now Remark 4 is valid in the setting of pro-p-groups; furthermore, the two "new versions" involving $f_{1}$ (of Remark 4) are equivalent by the preceding observation. It follows that Theorems 1 and 2 can be derived from their analogues in pro-p-groups (and vice versa).

\section{REFERENCES}

1. E. Artin, Geometric algebra, Interscience, New York, 1957. MR 18, 553.

2. J. Shapiro and J. Sonn, Free factor groups of one-relator groups, Duke Math J. (to appear).

3. A. Steinberg, On free nilpotent quotient groups, Math. Z. 85 (1964), 185196. MR 33 \# 176.

4. J. -P. Serre, Cohomologie galoisienne, 2nd ed., Lecture Notes in Math., vol. 5, Springer-Verlag, Berlin and New York, 1964. MR 31 \#4785.

5. J. Labute, Classification of Demushkin groups, Canad. J. Math 19 (1967), 106-132. MR 35 \#1674.

TECHNION, ISRAEL INSTITUTE OF TECHNOLOGY, HAIFA, ISRAEL 